\title{
Effect of blueberry feeding on plasma lipids in pigs
}

\author{
W. Kalt ${ }^{1}$, Kim Foote ${ }^{2}$, S. A. E. Fillmore ${ }^{1}$, Martha Lyon $^{3}$, T. A. Van Lunen ${ }^{4}$ and K. B. McRae \\ ${ }^{1}$ Agriculture and Agri-Food Canada, Atlantic Food and Horticulture Research Center, 32 Main Street, Kentville, \\ Nova Scotia B4N 1J5, Canada \\ ${ }^{2}$ Charlottetown Veterinary Clinic, 5 Kirkwood Drive, Charlottetown, PE C1A 2T4, Canada \\ ${ }^{3}$ Alberta Children's Hospital, Calgary Laboratory Services, Room B3-724, 2888 Shaganappi Trail NW, Calgary, \\ AB Т3B 6A8, Canada \\ ${ }^{4}$ Canadian Forestry Service - Atlantic Forestry Centre, PO Box 960, Corner Brook, NF A2H 6H3, Canada \\ (Received 16 April 2007 - Revised 4 October 2007 - Accepted 26 October 2007 - First published online 17 December 2007)
}

Two feeding trials were conducted with pigs to determine the effects of blueberry supplementation on plasma lipid levels and other indices of cardiovascular benefit. In the first trial, where basal diets contained a high level of plant-based components ( $70 \%$ soya, oats and barley), supplementation with 1, 2 and $4 \%$ blueberries resulted in a decrease in total, LDL- and HDL-cholesterol. The greatest reduction was observed in the $2 \%$ blueberry-fed pigs, where total, LDL- and HDL-cholesterol were reduced $11.7,15.1$ and $8.3 \%$, respectively. In the second trial where basal diets contained only $20 \%(\mathrm{w} / \mathrm{w}$ ) of soya, oats and barley, the lipid-modulating effect of blueberries was attenuated, so that supplementation with $1.5 \%$ blueberries reduced total cholesterol by $8 \%$, which occurred only in pigs whose diets had been supplemented with cholesterol ( $0.08 \%)$, $\mathrm{NaCl}(0.11 \%)$ and fructose $(9 \%)$. In the first feeding trial, blueberry supplementation had no effect on blood platelet activity. Blueberry supplementation also had no effect on the susceptibility of leucocyte DNA to oxidation in the first trial and no effect on the susceptibility of LDL to oxidation in the second trial. Results of these two feeding trials are discussed in relation to the effects of basal diet composition on lipid-modulating effects of blueberries.

Blueberries: Plasma lipids

Epidemiological evidence indicates that fruit, vegetable and whole-grain consumption is associated with a decreased risk of $\mathrm{CHD}^{(1)}$ and stroke ${ }^{(2)}$. Currently, specific fruit, vegetable and whole-grain components are being studied to determine the mechanisms underlying the health-protective properties of these foods. Of great interest for their possible cardioprotective effects are the (poly)phenolics, which are ubiquitous in plant foods and particularly abundant in berry crops $^{(3)}$. Results from in vitro and in vivo studies suggest that phenolics and, in particular, flavonoids, may benefit cardiovascular health in several ways. Flavonoids may act as antioxidants to inhibit LDL oxidation, and thereby protect against vascular insult by atherogenic oxidised $\mathrm{LDL}^{(4)}$. Flavonoids may reduce vascular inflammation related to atherosclerosis ${ }^{(5)}$. They are also reported to inhibit platelet aggregation and adhesion $^{(6)}$. Flavonoids may affect reverse cholesterol transport to decrease total and LDL-cholesterol (for a review, see $\left.\operatorname{Reed}^{(7)}\right)$. Some phenolic-containing foods, such as citrus ${ }^{(8)}$, red grape products ${ }^{(9)}$, tea ${ }^{(10)}$ and chocolate ${ }^{(11,12)}$ have been shown to affect cardiovascular attributes including those mentioned above.

Blueberries (BB) are of interest in health and nutritional science because of their high phenolic content compared with other fruit crops ${ }^{(13)}$. BB are relatively rich in anthocyanin flavonoids, chlorogenic acid, as well as proanthocyanidins ${ }^{(13)}$. They also contain abundant glucose, fructose and organic acids (especially citric and malic acid), starch, pectin, cellulose and other polysaccharides ${ }^{(14)}$. BB have been noted for their neuroprotective effects when studied in in vivo models of inflammation, oxidative stress, ageing and neurodegenerative disease (for a review, see Joseph et al. ${ }^{(15)}$ ) as well as in neural transplantation ${ }^{(16)}$.

Pigs are a suitable model for the study of dietary effects on human cardiovascular attributes, including plasma lipid composition. Pigs are omnivores and will eat essentially all foods common in the human diet. They have levels of LDL similar to humans and, like humans, are susceptible to diet-induced vascular disease. They have a propensity to develop atherosclerotic plaques at the same sites as humans (for example, aorta, carotid artery) and can undergo plaque regression. They have similar haemodynamic parameters, such as blood pressure, pulse pressure and heart rate (for reviews, see Turk \& Laughlin $^{(17)}$ and Moghadasian et al. $\left.{ }^{(18)}\right)$. Their platelets behave in a similar manner to those of humans ${ }^{(19)}$, and they have a similar body weight as humans. 
Soya, oats and barley contain components recognised for their lipid-modulating properties. Oats and barley contain $\beta$-glucan, which is purported to reduce cholesterol by increasing the hepatic conversion of cholesterol to bile acids ${ }^{(20)}$. Because of its gelling properties, $\beta$-glucan may also increase the viscosity of food in the lumen of the gut to create an unstirred layer at the intestinal mucosa which reduces absorption of food components, including cholesterol ${ }^{(20)}$. Soya protein and isoflavones have both been reported to have beneficial effects on serum lipids, although the relative importance of each component remains controversial ${ }^{(20)}$.

Because BB are a rich source of phenolics ${ }^{(13)}$ that are potentially cardioprotective ${ }^{(3,5-7)}$, the purpose of the present study was to determine whether $\mathrm{BB}$ feeding could affect plasma lipids, platelet aggregation, and blood coagulation and antioxidant indices. Two separate BB feeding trials are reported here; these two trials did not share a common treatment. Since the basal diet composition in the two feeding trials differed substantially in their content of soya, oats and barley, these two trials may provide an opportunity to examine the effect of basal diet composition, and $\mathrm{BB}$, on plasma lipids.

\section{Experimental methods}

Animals

These studies were conducted with the approval of the University of Prince Edward Island Animal Care Committee, under the Guidelines for the Care and Use of Experimental Animals (Canadian Council on Animal Care). Two pig feeding trials, trial 1 (T1) and trial 2 (T2), were performed in 2004 and 2005, respectively.

In both $\mathrm{T} 1$ and $\mathrm{T} 2$, healthy male castrated pigs (Yorkshire $\times$ Landrace) were obtained from a local producer at approximately age $32-41 \mathrm{~d}, 2$ weeks after weaning. Pigs, which weighed $15 \pm 2 \mathrm{~kg}$ at the beginning of the study, were housed in groups of five (T1) or six (T2), in pens that were $1.52 \mathrm{~m}$ wide, $3.04 \mathrm{~m}$ deep and $1.07 \mathrm{~m}$ high. After an acclimation period of $2 \mathrm{~d}$ (T1) or 2 weeks (T2), in which they were fed control diets, pigs were switched to experimental diets that had added BB (T1 and T2) and cholesterol, salt and fructose (T2) (Table 1). In both T1 and T2, pigs consumed food ad libitum, except that feed was removed from pens approximately $18 \mathrm{~h}$ before blood sampling (just before euthanasia at 4 and 8 weeks in T1, and 12 weeks in T2). Weight gain was measured weekly in individual pigs. Feed intake was measured weekly as a mean value for each pen.

In T1, there were two pens per experimental diet group, with five pigs per pen, at the beginning of the study. In T1, five pigs in each diet group (i.e. one pen) were euthanised after 4 weeks, and five pigs in another pen were euthanised after 8 weeks, for studies on anthocyanin bioavailability which will be reported elsewhere. In T2, the six pigs per diet group were euthanised after 12 weeks.

\section{Diets}

Basal diets were formulated to meet or exceed nutrient requirements of pigs weighing between 15 and $75 \mathrm{~kg}^{(21)}$. Basal diets were supplemented with whole freeze-dried, powdered BB (Vaccinium corymbosum L., var. 'Jersey') where indicated (Table 1). In T1, BB powder was added to diets at a concentration of $0,1,2$ and $4 \%(\mathrm{w} / \mathrm{w})$ and in T2 at $1.5 \%$ (w/w). To normalise the sugar concentration in T1 diets, glucose and fructose were present as BB sugar and/or added sugar, at a concentration of 12 and $13 \mathrm{~g} / \mathrm{kg}$ diet, respectively. Similarly in T2, glucose and fructose was added to the non-BB diets at a concentration of 4.95 and $4.5 \mathrm{~g} / \mathrm{kg}$ diet, respectively (Table 1). BB contain very small amounts of sucrose ${ }^{(22)}$ and diets were not normalised for BB sucrose. In both $\mathrm{T} 1$ and $\mathrm{T} 2$, diets were isoenergetic. Total energy was about $18 \%$ greater in $\mathrm{T} 1$ than $\mathrm{T} 2$. In both $\mathrm{T} 1$ and $\mathrm{T} 2$, diets were

Table 1. Composition of experimental diets

\begin{tabular}{|c|c|c|c|c|c|c|c|c|}
\hline \multirow{2}{*}{$\frac{\text { Ingredient (g/kg diet) }}{\text { Blueberry powder }}$} & \multicolumn{4}{|c|}{ Trial 1} & \multicolumn{4}{|c|}{ Trial 2} \\
\hline & 0 & 10 & 20 & 40 & 0 & 15 & 0 & 15 \\
\hline Fructose & 13 & 10 & 7 & 0 & 4.5 & 0 & 94.5 & 90 \\
\hline Salt & 0.29 & 0.29 & 0.29 & 0.29 & 0.29 & 0.29 & 1.42 & 1.42 \\
\hline Cholesterol from lard & 0 & 0 & 0 & 0 & 0 & 0 & 0.073 & 0.073 \\
\hline Soya meal (48\%) & 391 & 391 & 391 & 391 & $78 \cdot 2$ & $78 \cdot 2$ & $78 \cdot 2$ & $78 \cdot 2$ \\
\hline Barley & 200 & 200 & 200 & 200 & 40 & 40 & 40 & 40 \\
\hline Oats & 100 & 100 & 100 & 100 & 20 & 20 & 20 & 20 \\
\hline Rapeseed meal & 75 & 75 & 75 & 75 & 15 & 15 & 15 & 15 \\
\hline Fat Pak 100 & 75 & 75 & 75 & 75 & $66 \cdot 5$ & $66 \cdot 5$ & $66 \cdot 5$ & $66 \cdot 5$ \\
\hline Soya oil & 63 & 63 & 63 & 63 & $54 \cdot 5$ & $54 \cdot 5$ & $54 \cdot 5$ & 54.5 \\
\hline Corn \#2 $8 \%$ & 24 & 24 & 24 & 24 & $4 \cdot 8$ & $4 \cdot 8$ & $4 \cdot 8$ & $4 \cdot 8$ \\
\hline Calcium carbonate granules (limestone) & 12 & 12 & 12 & 12 & 0 & 0 & 0 & 0 \\
\hline Dicalcium phosphate (Biofos) & 11 & 11 & 11 & 11 & $2 \cdot 7$ & $2 \cdot 7$ & $2 \cdot 7$ & $2 \cdot 7$ \\
\hline Bran (22.7 kg bags) & 15 & 11 & 7 & 0 & 0 & 0 & 0 & 0 \\
\hline Gold Swine Ultra $(2 \mathrm{~kg} / \mathrm{T})$ & 2 & 2 & 2 & 2 & 2 & 2 & 2 & 2 \\
\hline Lysine & 0.5 & 0.5 & 0.5 & 0.5 & 4 & 4 & 4 & 4 \\
\hline Choline chloride $70 \%$ & 0.4 & 0.4 & 0.4 & 0.4 & 0.4 & 0.4 & 0.4 & 0.4 \\
\hline Meat and bone meal & 0 & 0 & 0 & 0 & $69 \cdot 1$ & $69 \cdot 1$ & $69 \cdot 1$ & $69 \cdot 1$ \\
\hline Herring meal & 0 & 0 & 0 & 0 & 135.5 & 135.5 & 135.5 & $135 \cdot 5$ \\
\hline Rice & 0 & 0 & 0 & 0 & 390 & 390 & 390 & 390 \\
\hline Cellulose & 0 & 0 & 0 & 0 & $95 \cdot 82$ & $95 \cdot 82$ & $95 \cdot 82$ & $95 \cdot 82$ \\
\hline
\end{tabular}


normalised for their amino acid content. Soya, oats and barley made up $70 \%(\mathrm{w} / \mathrm{w})$ of the diet in $\mathrm{T} 1$ and $20 \%$ of the diet in $\mathrm{T} 2$ (Table 1). In T2, cholesterol (0.08\%), $\mathrm{NaCl}(0.11 \%)$ and fructose $(9 \%)$ were added to diets that either did, or did not, contain $1.5 \%$ BB (Table 1 ).

The most abundant dietary antioxidant that was varied in $\mathrm{T} 1$ and $\mathrm{T} 2$ was the phenolics present in the BB powder. The next most abundant antioxidant present in the BB powder was vitamin $\mathrm{C}$, which contributes less than $5 \%$ to the total antioxidant capacity of $\mathrm{BB}^{(23)}$. The phenolic concentration of the $\mathrm{BB}$ powder was measured in extracts prepared both in water and in a solvent mixture of $40 \%$ acetone, $40 \%$ methanol, $20 \%$ water and $0.1 \%$ formic acid ${ }^{(13)}$. Colorimetric assays were used to measure the phenolic ${ }^{(24)}$ and anthocyanin ${ }^{(25)}$ content of the BB powder. Total phenolic and anthocyanin content were also measured using HPLC and diode array detection at 280 and $520 \mathrm{~nm}$, respectively ${ }^{(13)}$.

\section{Blood collection and analysis}

A blood sample was collected at 4 and 8 weeks in T1, and at $0,4,8$ and 12 weeks in $\mathrm{T} 2$, from the jugular vein of ketaminesedated pigs $(20 \mathrm{mg} / \mathrm{kg}$ body weight; intramuscular). Blood was collected into Vacutainer tubes (VWR International, Mississauga, ON, Canada) which contained specific anticoagulants, where needed, for clinical analyses. In both $\mathrm{T} 1$ and T2, blood was collected for a complete blood count and serum lipid analysis.

Total, LDL- and HDL-cholesterol, and TAG were measured directly using enzyme-linked assays, and monitored using a Hitachi 911 auto-analyser (Roche, Laval, QU, Canada) and test kits obtained from Diagnostic Chemicals (Charlottetown, PE, Canada). For measurement purposes, HDL was solubilised from particles, and the reaction of LDL, VLDL and chylomicrons were selectively inhibited by their conversion into stable complexes. In LDL measurements, VLDL-, HDL- and chylomicron-cholesterol were oxidised before the solubilisation and measurement of LDL.

In T1, blood was also collected for the measurement of platelet function, prothrombin and activated partial thromboplastin time, and leucocyte DNA oxidisability. In T1 platelet function was analysed using the automated platelet function analyzer (Dade Behring, Mississauga, ON, Canada) according to the manufacturer's specifications. This instrument simulates in vitro the processes of platelet adhesion and aggregation following vascular injury. Freshly collected whole citrated blood and pre-measured collagen-adrenaline agonists contained in cartridges (Dade Behring) were mixed within the instrument. The time needed to form a platelet aggregate to occlude a capillary tube was determined. In T1, prothrombin time and activated partial thromboplastin time were measured in platelet-poor plasma obtained by centrifuging citrated blood. Automated prothrombin time and activated partial thromboplastin time determinations were carried out using a MLA Electra 1400C coagulation analyser (Beckman Coulter, Mississauga, ON, Canada) according to the manufacturer's specifications.

In T2, blood was also collected for LDL oxidisability and for the determination of plasma concentrations of C-reactive protein, insulin and glucose. Plasma levels of C-reactive protein were measured using an ELISA test kit specifically developed for swine (Immunology Consultants Laboratory,
Inc., Newberg, OR, USA). Plasma glucose and insulin were measured using diagnostic services provided by the Queen Elizabeth II Hospital (Charlottetown, PE, Canada). Insulin was measured using Immulite test kits (Diagnostic Products Corporation, Los Angeles, CA, USA).

\section{Antioxidant assays}

In T1, DNA oxidisability was measured according to the method of Batel et al. ${ }^{(26)}$ using leucocytes isolated from freshly collected blood and cryo-preserved at $-80^{\circ} \mathrm{C}$. Briefly, leucocytes were rapidly thawed and counted before they were treated with $250 \mu \mathrm{mol} / 1 \mathrm{H}_{2} \mathrm{O}_{2}$ in $\mathrm{PBS}$ for $5 \mathrm{~min}$ on ice. Leucocytes were centrifuged and the pellet was re-suspended and diluted to approximately 120 cells $/ \mu$ in $\mathrm{TE}$ buffer (10 mM-tri(hydroxymethyl)-aminomethane-HCl, $\mathrm{pH}$ 7.4, containing $1 \mathrm{mm-EDTA}$ ). Proteinase K (Sigma Chemical Co., Oakville, ON, Canada) in TE buffer was added to achieve a proteinase $\mathrm{K}$ concentration of $1 \mathrm{mg} / \mathrm{ml}$. Cells were allowed to rupture during $30 \mathrm{~min}$ at $37^{\circ} \mathrm{C}$. Samples $(25 \mu \mathrm{l})$ of ruptured cells, plus $25 \mu \mathrm{l}$ of a 50 -fold dilution of PicoGreen dye (Invitrogen, Burlington, ON, Canada) in TE buffer, were added to a black microwell plate (Cliniplate; VWR International, Mississauga, ON, Canada) for fluorescence measurement (excitation $475 \mathrm{~nm}$, emission at $520 \mathrm{~nm}$ ). After an initial measurement was made, $250 \mu \mathrm{l}$ of the DNA-denaturing mixture (TE$\mathrm{NaOH}$ buffer, pH 12.4) was added to the wells. Wells were read every $1 \mathrm{~min}$ for $40 \mathrm{~min}$. Calf thymus dsDNA (Bio-Rad, Montreal, QU, USA) diluted in TE buffer was used as a standard in the assay at $50 \mathrm{ng}$ per well. Data were calculated as the strand scission factor (SSF), with:

$$
\mathrm{SSF}=\log _{10}\left(\% \mathrm{dsDNA}_{(40 \mathrm{~min})} / \% \mathrm{dsDNA}_{(0 \mathrm{~min}-\text { blank })}\right) .
$$

To measure LDL oxidisability in T2, LDL was partially purified from previously frozen plasma using affinity chromatography on heparin-agarose (Sigma Chemical Co., Oakville, ON, Canada), as described by Vinson et al. ${ }^{(27)}$. After loading $1 \mathrm{ml}$ of plasma, the column was washed with water, then $0.7 \%$ $\mathrm{NaCl}$, and finally $2.7 \% \mathrm{NaCl}$. The fraction removed with $2.7 \% \mathrm{NaCl}$, which contained LDL and VLDL, was used in the lipid oxidation assay. The $2.7 \% \mathrm{NaCl}$ fraction was diluted to $2.6 \mathrm{~g}$ protein/l using the Bradford reagent (Sigma Chemical Co.). The LDL oxidation assay $(250 \mu \mathrm{l})$ was conducted in PBS, pH 7.4, and monitored at $234 \mathrm{~nm}$ using a microplate reader (SpectraMax 190; Molecular Devices, Sunnyvale, CA, USA). The uninhibited formation of conjugated dienes arising from lipid oxidation was taken as the inflection point in the $234 \mathrm{~nm}$ profile. It took a minimum of $50 \mathrm{~min}$ for an inflexion point to occur, indicating that the LDL was not extensively oxidised at the beginning of the assay.

\section{Statistical analysis}

Data were analysed using procedures in GenStat ${ }^{\circledR}$ (VSN International Ltd, Hemel Hempstead, Herts, UK) ${ }^{(28)}$. In T1 ANOVA was used to determine the effects of BB dose and feeding duration on the various attributes. In this analysis, pigs were the primary experimental units. Feeding duration (4 v. 8 weeks) was the secondary unit examined among the pigs that were in the study for 8 weeks. The ANOVA table was partitioned to report variation among and within 
pigs. The overall effects of $\mathrm{BB}$ dose and pens (i.e. two pens per BB diet group at 4 weeks) and their interaction were assessed from the variation among pigs. The effects of feeding duration and its interaction with $\mathrm{BB}$ diets were assessed within the pigs that were fed for up to 8 weeks. Differences among the $\mathrm{BB}$ diets, and their interaction with feeding duration, were assessed with orthogonal contrasts. These included BBsupplemented $v$. non-BB-supplemented diets, and BB diets containing $4 \% v .1$ and $2 \% \mathrm{BB}$, and $2 \%$ v. $1 \% \mathrm{BB}$. The initial value for each blood attribute was used as a covariate for each pig in the analysis.

In T2, blood attributes were measured after $0,4,8$ and 12 weeks of feeding. The mean value and the regression slope of the change over the 12-week feeding period was calculated, and analysed by ANOVA and by a procedure using a $2 \times 2$ factorial design ( $\pm \mathrm{BB}, \pm$ cholesterol, salt and fructose). The initial (week 0) value for each blood attribute was used as a covariate for each pig in the analysis.

\section{Results}

Diets

Analysis of the BB powder revealed different contents of phenolics and anthocyanins depending on whether the $\mathrm{BB}$ powder was extracted in water or solvent. Colorimetric measurement of total phenolics in water and solvent-based extracts indicated a content of 6.64 and $13.4 \mathrm{mg}$ gallic acid equivalents/g DM BB powder, respectively. Colorimetric measurement of anthocyanin content in water and solventbased extracts indicated a content of 1.57 and $7.97 \mathrm{mg}$ cyanidin 3-glucoside equivalents/g DM BB powder, respectively. HPLC analysis of the BB powder (Fig. 1) shows the profile of total phenolics $(280 \mathrm{~nm})$ and of anthocyanins $(520 \mathrm{~nm})$ in the 'Jersey' BB powder extracted in solvent (Fig. 1 (a) and (b)) or water (Fig. 1 (c) and (d)). The major non-anthocyanin phenolic omponent was chlorogenic acid, which was determined based on retention time in comparison with a pure standard.

\section{Feed intake and weight gain}

In $\mathrm{T} 1$ there was no difference in feed intake (average $\mathrm{kg}$ feed consumed/pig per d) among the diet groups in T1 (Fig. 2). However, weight gain was affected by BB dose $(P=0.023)$. While weight gain was higher in the $1 \% \mathrm{BB}$-fed pigs it was lower in the $4 \%$ BB-fed pigs, compared with the control $(0 \%$ BB). Control and $2 \%$ BB-fed pigs had a similar weight gain (Fig. 2).

In $\mathrm{T} 2$, there was no significant difference $(P=0.774)$ among the diet groups in feed intake ( $\mathrm{kg}$ consumed/pig per $\mathrm{d}$ ), nor for weight gain related to either the addition of $1.5 \%$ BB $(P=0.419)$, or cholesterol, salt and fructose (CSF) $(P=0 \cdot 162)$, or both BB and CSF $(P=0 \cdot 101)$.

\section{Blood lipids}

In $\mathrm{T} 1$, plasma lipid concentrations were not significantly different when measured in the same pigs at 4 and 8 weeks; therefore results of these two time points (forty pigs at 4 weeks, and twenty pigs at 8 weeks) were combined
(Table 2). The plasma concentration of total, LDL- and HDL-cholesterol were lower in pigs that received $\mathrm{BB}$ in the T1 basal diet that contained $70 \%(\mathrm{w} / \mathrm{w})$ of soya, oats and barley (Fig. 3). In T1 the addition of BB lowered total cholesterol $(P=0.004)$, LDL $(P<0.001)$ and HDL $(P=0.022)$. Total cholesterol appeared to decline in relation to $\mathrm{BB}$ dose between 0,1 and $2 \%$ of $\mathrm{BB}$, but was similar between 2 and $4 \% \mathrm{BB}$ (Fig. 3). The $2 \% \mathrm{BB}$ dosage was the most effective in lowering total cholesterol $(-11.7 \%)$ (Fig. 3), LDL $(-15.1 \%)$ and HDL-cholesterol $(-8.3 \%)$ (Fig. 4). Compared with $2 \% \mathrm{BB}$, total and LDL-cholesterol increased in the $4 \%$ BB-fed pigs, albeit not significantly (Figs. 3 and 4).

In $\mathrm{T} 2$, where basal diets contained $20 \%$, instead of $70 \%$ (w/w), of soya, oats and barley, the addition of CSF to the diets resulted in higher total $(P<0.001)$ and LDL plasma cholesterol $(P<0 \cdot 001)$ (Table 3). Although BB feeding resulted in a trend $(P=0.043)$ to lower total plasma cholesterol over the 12-week feeding period, the lipid-modulating effect of $\mathrm{BB}$ was attenuated compared with $\mathrm{T} 1$. The lipid-lowering effect of $\mathrm{BB}$ appeared to be limited to pigs whose diets were supplemented with CSF (Fig. 5) although there was no significant interactive effect between BB and CSF (Table 3).

\section{Blood clotting}

Blood clotting attributes were measured in T1 only. There was no effect of BB feeding on platelet activation when measured ex vivo using collagen-adrenaline as the agonist and an automated platelet function analyser $(P=0 \cdot 194)$. Similarly, other clotting parameters including plasma prothrombin time and activated partial thromboplastin time were not affected by BB feeding ( $P=0.097$ and $P=0.081$, respectively).

\section{Antioxidant and other effects}

There was no effect of BB supplementation on the oxidisability of either leucocyte DNA in T1 $(P=0.968)$ or LDL oxidisability in T2 $(P=0.457)$. Similarly, there was no effect of 12 weeks of $\mathrm{BB}$ supplementation on plasma concentration of C-reactive protein, insulin or fasting glucose in T2 (data not shown).

\section{Discussion}

Pigs are known to be a useful model for the study of human cardiovascular attributes ${ }^{(18)}$ in human health research. The 1 to $4 \%(\mathrm{w} / \mathrm{w}) \mathrm{BB}$ powder contents in the diet, in relation to feed intake of pigs, were approximately equivalent to 1-4 one-cup servings of BB per $\mathrm{kg}$ diet, based on anthocyanin content. One cup of fresh BB weighs approximately $125 \mathrm{~g}$. Therefore, in the present study, normal healthy pigs were fed BB doses that could be reasonably achieved in the adult human diet, and suggests that the observed effect from BB supplementation could occur in healthy humans.

\section{Diets}

The phenolic components of the BB powder were up to five times less abundant in a water extract compared with the solvent extract (Fig. 1). The extraction of phenolics and anthocyanins into water (Fig. 1 (c) and (d)) may simulate more 
(a)

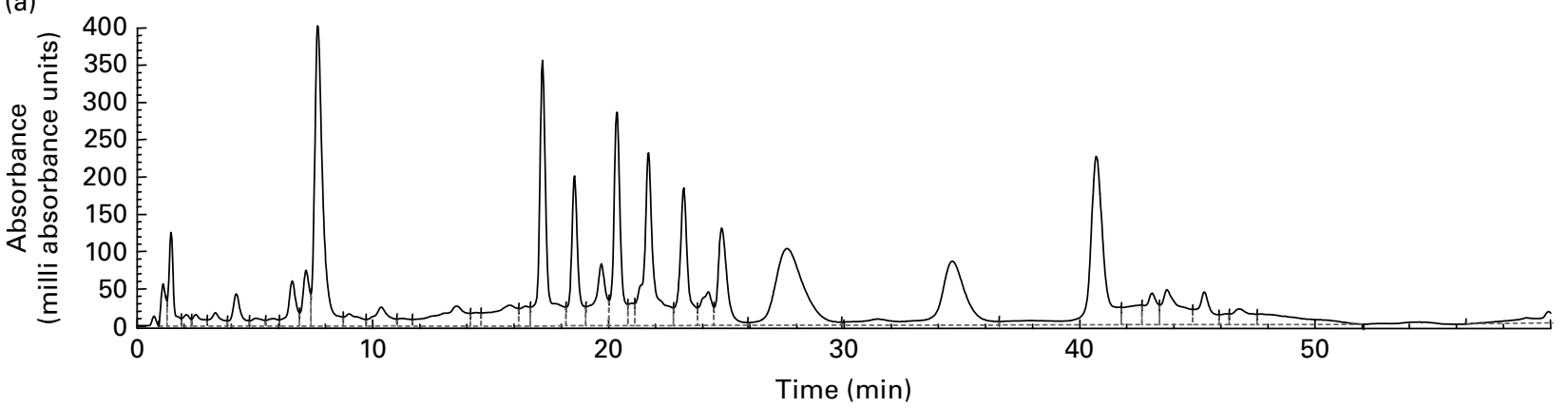

(b)

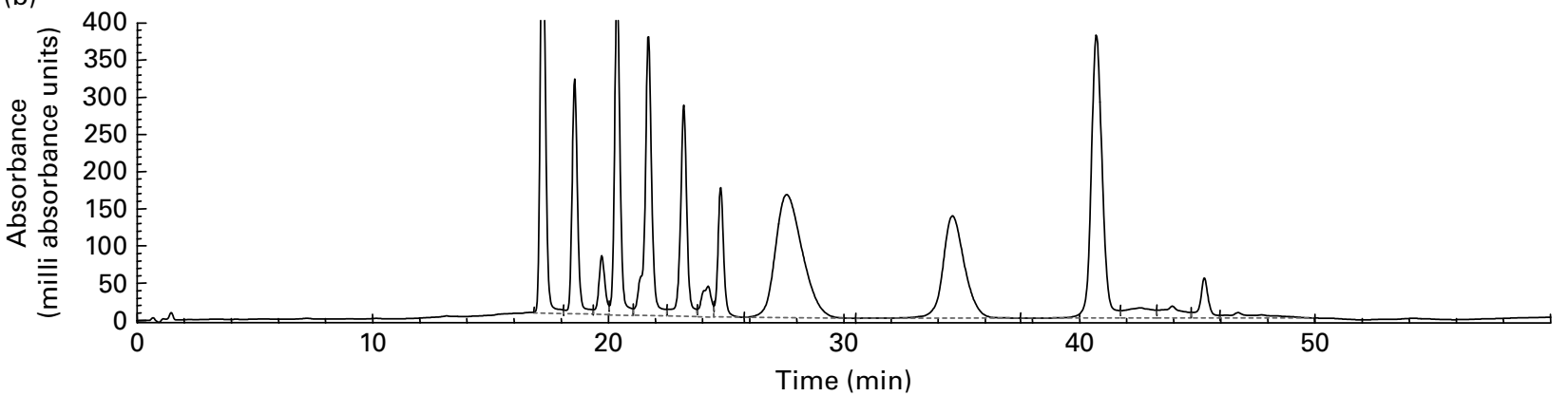

(c)

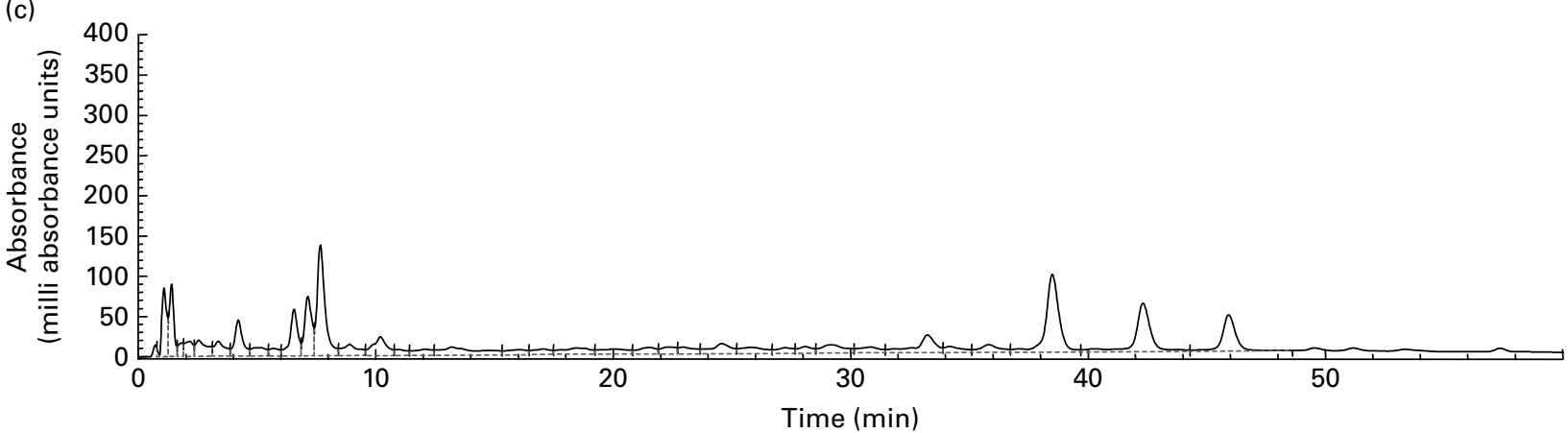

(d)

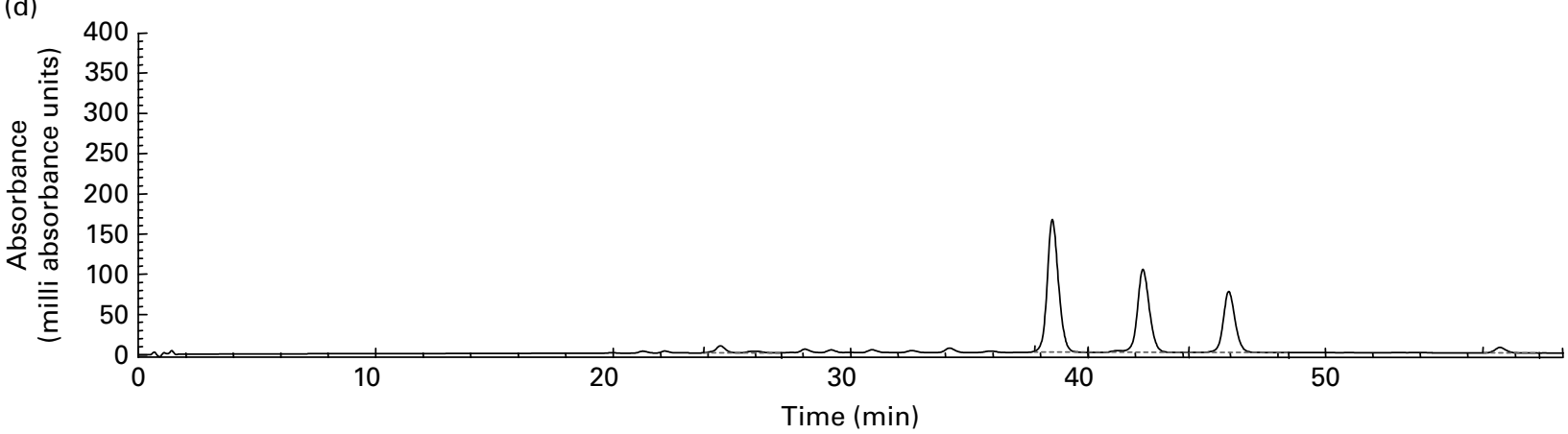

Fig. 1. HPLC profiles of freeze-dried blueberry powder, indicating total phenolic components at $280 \mathrm{~nm}(-)$ and anthocyanins at $520 \mathrm{~nm}(---)$, for samples extracted in acetone-methanol-water-formic acid (40:40:20:0.1, by vol.) (a,b) and water (c,d).

closely the extraction of phenolics from the BB and food matrix during normal mastication and the early stages of digestion. It is unlikely that the release of $\mathrm{BB}$ phenolics and anthocyanins during eating would be as effective as their release during solvent extraction (Fig. 1 (a) and (b)), particularly since phenolic components (including anthocyanins) are known to bind to various macromolecules, especially protein.
In both $\mathrm{T} 1$ and $\mathrm{T} 2$, fat provided approximately $30 \%$ of the energy in the diet (Table 1). In T2, we simulated a poor 'heart health' diet by including a lower level of plant-based foods than in T1, and added CSF that is known to increase plasma lipids $^{(29)}$, blood pressure ${ }^{(30)}$ and blood glucose ${ }^{(31)}$, respectively. All of these factors can contribute adversely to cardiovascular health. 


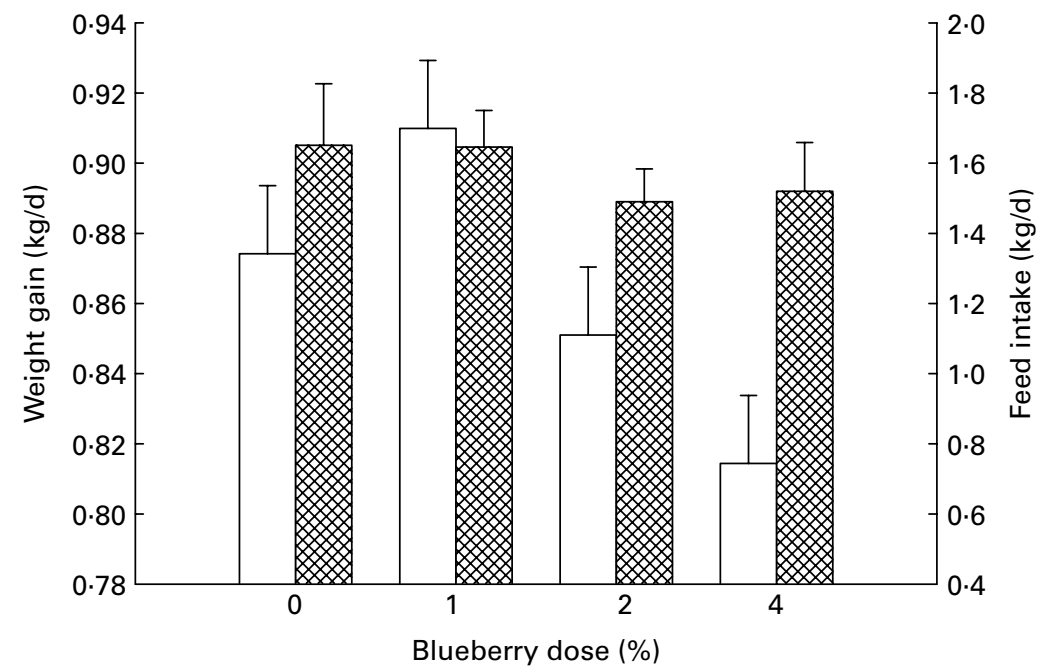

Fig. 2. Rate of weight gain ( $\square$ ) and feed intake (图) for pigs in trial 1. Weight gain was measured weekly in individual pigs. Feed intake was measured weekly as a single value for five pigs (i.e. one pen). Values are means, with their standard errors represented by vertical bars.

\section{Weight gain}

In $\mathrm{T} 1, \mathrm{BB}$ affected the weight gain of pigs, where $4 \% \mathrm{BB}$ reduced it, while 1 and $2 \% \mathrm{BB}$ did not affect it significantly. This result is interesting because recent studies report effects of anthocyanins on weight gain. Tsuda et al. ${ }^{(32)}$ showed that purple maize anthocyanins reduced weight gain in mice on high-fat diets, and subsequently showed that purple maize anthocyanins can affect adipocyte cytokine secretion in vitro ${ }^{(33)}$. In their 2003 study, Tsuda et al. supplemented mouse diets with $2 \mathrm{mg}$ per $\mathrm{g}$ feed, while our $4 \%$ pig diet contained only $0.312 \mathrm{mg}$ cyanidin 3 -glucoside equivalents per $\mathrm{g}$ feed. Therefore, the results of the present study support the observations of Tsuda et al. ${ }^{(32)}$ but were observed at a much lower level of dietary anthocyanin. Tsuda et al. ${ }^{(32)}$ also reported that purple maize anthocyanins normalised glucose, insulin and leptin concentrations increased by the high-fat diet. Jayaprakasam et al. ${ }^{(34)}$ reported that anthocyanins affected insulin release in isolated pancreatic $\beta$-cells in vitro. The lack of a significant effect of feeding $\mathrm{BB}$ on

Table 2. Trial 1 plasma lipid concentrations and probability values for additions of whole blueberry (BB) powder in the diet measured after 4 and 8 weeks of feeding ${ }^{\star}$

\begin{tabular}{lcccc}
\hline & \multicolumn{4}{c}{ Plasma lipid (mmol/l) } \\
\cline { 2 - 5 } BB dose & TC & LDL & HDL & TAG \\
\hline $0 \%$ & 2.48 & 1.18 & 1.15 & 0.61 \\
$1 \%$ & 2.33 & 1.05 & 1.11 & 0.69 \\
$2 \%$ & 2.22 & 0.99 & 1.05 & 0.79 \\
$4 \%$ & 2.27 & 1.02 & 1.04 & 0.70 \\
Mean & 2.33 & 1.06 & 1.09 & 0.70 \\
SEM & 0.061 & 0.030 & 0.032 & 0.058 \\
F probability & & & & \\
$\quad 0 \%$ v. 1, 2, 4\% BB & 0.01 & $<0.001$ & 0.04 & 0.12 \\
BB $\times$ time & 0.48 & 0.75 & 0.35 & $0.002 \dagger$ \\
\hline
\end{tabular}

TC, total cholesterol; time, 4 and 8 weeks

* Trial 1 diets contained $70 \%(\mathrm{w} / \mathrm{w})$ soya, oats and barley.

†TAG at 4 weeks was $0.66,0.80,0.75$ and $0.73 \mathrm{mmol} / \mathrm{l}$ and TAG at 8 weeks was $0.49,0.47,0.88$ and $0.64 \mathrm{mmol} / \mathrm{l}$, for $0,1,2$ and $4 \% \mathrm{BB}$, respectively. plasma insulin in $\mathrm{T} 2$, as compared with the in vitro results reported by Jayaprakasam et al. ${ }^{(34)}$, may be partially due to the low in vivo bioavailability of anthocyanins ${ }^{(35)}$.

\section{Plasma lipids}

The present study is the first to report hypolipidaemic effects of $\mathrm{BB}$, and is interesting particularly since the effects in $\mathrm{T} 1$ and $\mathrm{T} 2$ were observed at moderate $\mathrm{BB}$ doses. In $\mathrm{T} 1$, the reduction in total and LDL-cholesterol was a desirable result of $\mathrm{BB}$ supplementation, while the reduction in HDL and the lack of an effect on plasma TAG level was not.

Other phenolic-containing diet supplements have also been reported to affect plasma lipids. Citrus flavones and flavonones isolated from tangerine peel reduced total and LDL, and TAG, but did not affect HDL-cholesterol in hamsters with diet-induced hypercholesterolaemia ${ }^{(8)}$. Since these components originated from a non-food source (i.e. peel) it is not possible to compare a serving size with the $\mathrm{BB}$ doses employed here. A polyphenol extract obtained from a mixture of red, purple and green grapes, incorporated into diets at the approximate equivalent of $56 \mathrm{~g}$ fresh grapes per $100 \mathrm{~g}$ diet, decreased plasma TAG and VLDL in ovariectomised

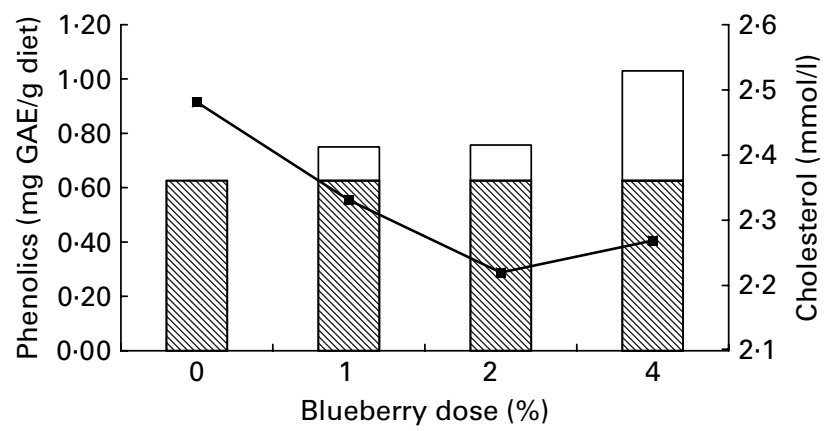

Fig. 3. Trial 1 total plasma cholesterol (-ם-) in pigs measured after both 4

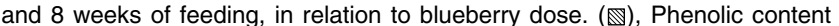
of the basal diet; $(\square)$, blueberry phenolic content of the diet; GAE, gallic acid equivalents. SEM 0.011 for cholesterol concentration. 


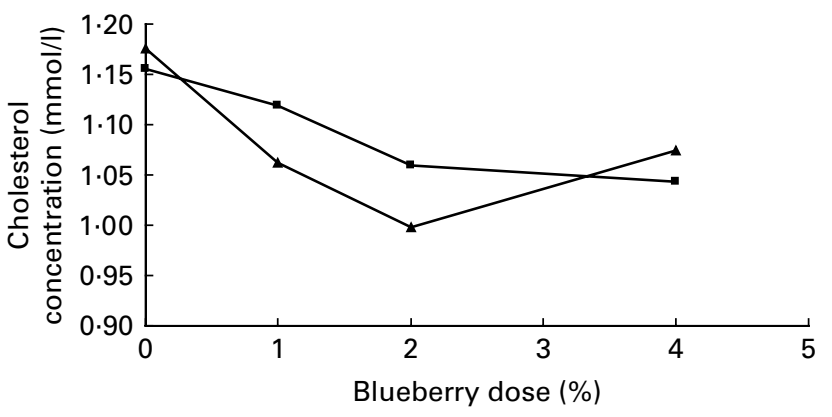

Fig. 4. Trial 1 plasma LDL- $(\boldsymbol{\Delta})$ and HDL-cholesterol $(\boldsymbol{\square})$ in pigs measured after both 4 and 8 weeks of feeding, in relation to blueberry phenolic dose. SEM 0.037 for LDL-cholesterol concentration; SEM 0.040 for HDL-cholesterol concentration.

guinea-pigs fed cholesterol-enriched diets ${ }^{(9)}$. VLDL concentration was not measured in the present study.

\section{Lipid modulation by blueberries in trial 1 and trial 2}

Although the two feeding trials are not directly comparable because they lacked a common treatment group, the results suggest that the lipid-modulating effects of $\mathrm{BB}$ in $\mathrm{T} 1$ and T2 may have been influenced by the composition of the basal diet. In T1, where BB had the greatest cholesterol-lowering effect, basal diets were rich in plant foods, including soya, oats and barley (Table 1). Cholesterol-lowering effects have been reported for each of the major components of the T1 basal diets, i.e. soya, oats and barley (for a review, see Kerckhoffs et al. $\left.{ }^{(20)}\right)$. In T2, however, where the combined content of soya, oats and barley was reduced from $70 \%$ (w/ w) to $20 \%(\mathrm{w} / \mathrm{w})$, feeding $1.5 \% \mathrm{BB}$ had a reduced effect on plasma cholesterol. The maximum plasma cholesterol reduction effect was only $8 \%$ in T2, observed after feeding for 12 weeks with $1.5 \%$ BB (Fig. 5), while it was $12 \%$ lower after feeding $2 \% \mathrm{BB}$ in $\mathrm{T} 1$ (Fig. 4) for either 4 or 8 weeks. It is possible that in T1, the higher levels of cholesterol-lowering components worked more effectively in combination with $\mathrm{BB}$ to beneficially affect plasma lipid levels in the pigs. The trends in plasma lipid modulation by BB may have been more apparent if there had been greater replication in the diet treatments; in T1 $n 5$ and in T2 $n 6$.

The observation in $\mathrm{T} 2$ that $\mathrm{BB}$ appeared to only affect plasma cholesterol in pigs fed poor 'heart health' diets

Table 3. Trial $2 F$ probability values for changes in plasma lipids over time $(0,4,8$ and 12 weeks) in relation to diets with and without $1.5 \%$ blueberries, or cholesterol, salt and fructose*

\begin{tabular}{lccc}
\hline & \multicolumn{3}{c}{ F probability } \\
\cline { 2 - 4 } & CSF† & Blueberries $\neq$ & CSF $\times$ blueberries \\
\hline Total cholesterol & $<0.001$ & 0.043 & NS \\
HDL & NS & NS & NS \\
LDL & $<0.001$ & NS & NS \\
TAG & NS & NS & NS \\
\hline
\end{tabular}

CSF, cholesterol, salt and fructose.

* Trial 2 basal diets contained $20 \%(\mathrm{w} / \mathrm{w})$ soya, oats and barley.

† Added cholesterol $(0.08 \%), \mathrm{NaCl}(0.11 \%)$ and fructose $(9.0 \%)$.

$\$ 1.5 \%$ whole blueberry powder. (i.e. added CSF) (Fig. 5) supports other observations that lipid modulation by food components is most effective in models of diet- or genetically induced hyperlipidaemia. For example, cranberries only reduced cholesterol in genetically hypercholesterolaemic pigs, and not in normal pigs ${ }^{(7)}$, and lipid-lowering effects of citrus were observed in hamsters with diet-induced hypercholesterolaemia ${ }^{(8)}$.

\section{Lack of effects on platelet activity and blood clotting}

While flavonoids, including anthocyanins, have been reported to reduce blood platelet activity in vitro and ex vivo ${ }^{(5,36)}$, and the propensity of clot formation ${ }^{(3)}$, no effect of $\mathrm{BB}$ on pig blood platelet activity was observed in the present study as measured with a Dade Behring platelet function analyzer (Dade Behring). Differences in methodology may explain this inconsistency. However, in conjunction with the present study, an informal evaluation of BB juice consumption on platelet function activity in twenty-three human volunteers was examined. The BB juice, which was consumed daily for 1 month and provided $900 \mathrm{mg}$ gallic acid equivalents of phenolic components, produced no effect on platelet activity (W Kalt, unpublished results). Anthocyanin effects on platelet aggregation were suggested by the results of Keevil et al. ${ }^{(6)}$ where purple grape juice, but not orange juice, reduced platelet aggregation in vitro. Hubbard et al. ${ }^{(37)}$ also failed to see an effect of a polyphenolic-rich diet on platelet function. The poor bioavailability of the anthocyanins ${ }^{(35)}$ may have contributed to the lack of a significant effect of BB observed in both pigs and human subjects.

\section{Conclusion}

Results of T1 and T2 suggest that BB may be another phenolic-rich food that can beneficially affect plasma lipid status. The two feeding trials illustrate how lipid-modulating dietary components (such as BB, soya, oats, and barley) may function synergistically to beneficially affect plasma lipid status. These results complement the work by Jenkins

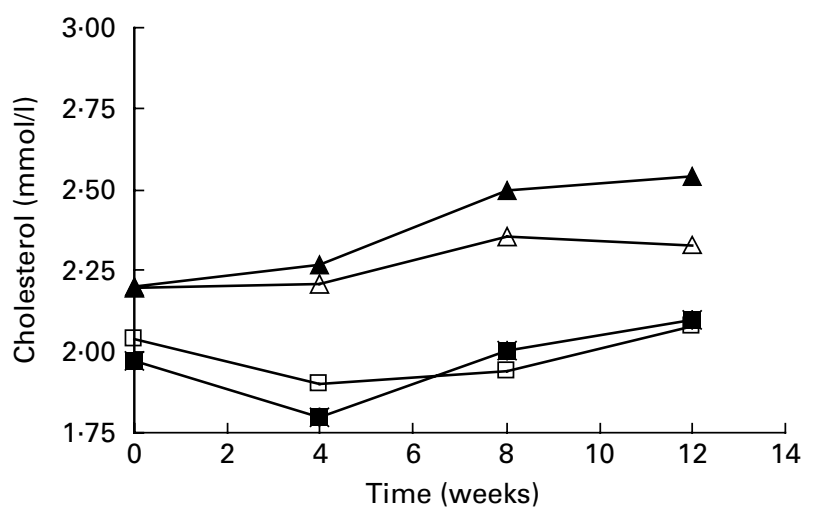

Fig. 5. Trial 2 total plasma cholesterol over a 12-week feeding period in pigs fed diets which contained either 0 or $1.5 \%$ blueberry powder: $(\Delta), 1.5 \%$ blueberry-supplemented diet supplemented with cholesterol $(0.08 \%), \mathrm{NaCl}$ $(0.11 \%)$ and fructose $(9.0 \%) ;(\square), 1.5 \%$ blueberry-supplemented diet not

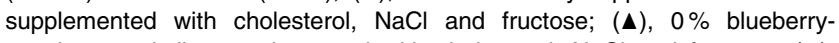
supplemented diet supplemented with cholesterol, $\mathrm{NaCl}$ and fructose; ( $\mathbf{\square})$, $0 \%$ blueberry-supplemented diet not supplemented with cholesterol, $\mathrm{NaCl}$ and fructose. SEM 0.0428 for total plasma cholesterol concentration. 
et al. ${ }^{(38)}$ on the 'Portfolio Diet'. Jenkins et al. ${ }^{(38)}$ have shown that the Portfolio Diet, which includes several cholesterollowering foods and food components (sterols, stanols, soluble fibre, and phenolics), can reduce plasma lipid levels, and can be as effective as statin drug treatment in doing so.

\section{Acknowledgements}

The authors are grateful to Jane McDonald, Melinda VinqvistTymchuk, Kristen Doncaster, Basil Dickson, and Karen Burgher-Maclellan of Agriculture and Agri-Food Canada (AAFC) and Alfred Mitchell of the Atlantic Veterinary College for their excellent technical assistance in maintenance and treatment of the pigs and in data collection and management. Financial support for the present study was provided to W. K. by the United States Highbush Blueberry Council and the Agriculture and Agri-Food Canada Matching Investment Initiative. Their support for the present study does not constitute an endorsement of their products and services over others.

\section{References}

1. Joshipura KJ, Hu FB, Manson JE, et al. (2001) The effect of fruit and vegetable intake on risk of coronary heart disease. Ann Intern Med 134, 1106-1114.

2. Fung TT, Stampfer MJ, Manson JE, Rexrode KM, Willett WC $\&$ Hu FB (2004) Prospective study of major dietary patterns and stroke risk in women. Stroke 35, 2014-2019.

3. Manach C, Mazur A \& Scalbert A (2005) Polyphenols and prevention of cardiovascular diseases. Curr Opin Lipidol 16, $77-84$.

4. Castellani W (2004) Metabolic and nutritional aspects of the atherogenic atypical lipoproteins: lipoprotein(a), remnant lipoproteins, and oxidized low-density lipoprotein. Nutr Res $\mathbf{2 4}$, 681-693.

5. Folts JD (2002) Potential health benefits from the flavonoids in grape products on vascular disease. In Flavonoids in Cell Function, pp. 95-111 [B Buslig and J Manthey, editors]. New York: Kluwer Academic/Plenum Publishing.

6. Keevil JG, Osman HE, Reed JD \& Folts JD (2000) Grape juice, but not orange juice or grapefruit juice, inhibits human platelet aggregation. J Nutr 130, 53-56.

7. Reed J (2002) Cranberry flavonoids, atherosclerosis and cardiovascular health. Crit Rev Food Sci Nutr 42, 301-316.

8. Kurowska EM \& Manthey JA (2004) Hypolipidemic effects and absorption of citrus polymethoxylated flavones in hamsters with diet-induced hypercholesterolemia. J Agric Food Chem 52, 2879-2886

9. Zern TL, West KL \& Fernandez ML (2003) Grape polyphenols decrease plasma triglycerides and cholesterol accumulation in the aorta of ovariectomized guinea pigs. J Nutr 133, $2268-2272$

10. Dufresne CJ \& Farnworth ER (2001) A review of latest findings on the health promotion properties of tea. J Nutr Biochem 12, 404-421.

11. Steinberg FM, Bearden MM \& Keen CL (2003) Cocoa and chocolate flavonoids: implications for cardiovascular health. $J \mathrm{Am}$ Diet Assoc 103, 215-223.

12. Kris-Etherton P \& Keen C (2002) Evidence for the antioxidant flavonoids in tea and cocoa are beneficial for cardiovascular health. Curr Opin Lipidol 13, 41-49.

13. Kalt W, MacKinnon S, McDonald J, Vinquist M, Craft C \& Howell A (2007) Phenolics of Vaccinium berries and other fruit crops. J Sci Food Agric (Epublication ahead of print version September 2007. http.//dx.doi.org/10.1002/jsfa.2991)

14. Eck P (1988) Blueberry Science, p. 81. New Brunswick, NJ: Rutgers University Press.

15. Joseph JA, Shukitt-Hale B \& Casadesus G (2005) Reversing the deleterious effects of aging on neuronal communication and behavior: beneficial properties of fruit polyphenolic compounds. Am J Clin Nutr 81, Suppl. 1, 313S-316S.

16. Willis L, Bickford P, Zaman V, Moore A \& Granholm A-C (2005) Blueberry extract enhances survival of neural transplants of intra-ocular hippocampal transplants. Cell Transplant 14, 213-223.

17. Turk JR \& Laughlin MH (2004) Physical activity and atherosclerosis: which animal model? Can J Appl Physiol 29, $657-683$

18. Moghadasian MH, Frolich JJ \& McManum BM (2001) Advances in experimental dyslipidemia and atherosclerosis. Lab Invest $\mathbf{8 1}$ $1173-1183$.

19. Goodman S (1999) Sheep, pig and human-material interactions with model cardiovascular materials. J Biomed Mater Res 45, 240-250.

20. Kerckhoffs DAJM, Brouns F, Hornstra G \& Mensink RP (2002) Effects on the human serum lipoprotein profile of $\beta$-glucan, soy protein and isoflavones, plant sterols and stanols, garlic and tocotrienols. J Nutr 132, 2494-2502.

21. Subcommittee on Swine Nutrition, Committee in Animal Nutrition \& National Research Council (1998) Nutrient Requirements of Swine, 10th revised ed. Washington, DC: National Academy Press.

22. Kalt W \& McDonald JE (1996) Chemical composition of lowbush blueberry cultivars. J Am Soc Hort Sci 121, 142-146.

23. Prior RL, Cao G, Martin A, et al. (1998) Antioxidant capacity is influenced by total phenolic and anthocyanin content, maturity and variety of Vaccinium species. J Agric Food Chem 46, $2686-2693$

24. Singleton VL \& Rossi JA (1965) Colorimetry of total phenolics with phosphomolybdic-phosphotungstic acid reagents. Am J Enol Vitic 16, 144-158.

25. Lee J, Durst RW \& Wrolstad RE (2005) Determination of total monomeric anthocyanin pigment content of fruit juices, natural colorants, and wines, by the $\mathrm{pH}$ differential method: collaborative study. J AOAC Int 88, 1269-1278.

26. Batel R, Jakšić Ž, Bihari N, Hamer B, Fafandel M, Chauvin C, Schröder HC, Müller WEG \& Zahn RF (1999) A microplate assay for DNA damage determination (Fast Micromethod) in cell suspensions and solid tissues. Anal Biochem 270, 195-200.

27. Vinson JA, Proch J \& Pratima B (1999) Determination of the quantity and quality of polyphenol antioxidants in foods and beverages. Methods Enzymol 335, 103-114.

28. Payne RW (2003) The Guide to GenStat ${ }^{\circledR}$ Release 7.1; Statistics. Oxford, UK: VSN International Ltd.

29. Kuller LH (2006) Cholesterol, lipid and cardiovascular disease. Nutr Rev 64, S15-S26.

30. Franco V \& Oparil S (2006) Salt sensitivity, a determinant of blood pressure, cardiovascular disease and survival. J Am Coll Nutr 25, 247S-255S.

31. Gaby AR (2005) Adverse effects of dietary fructose. Altern Med Rev 10, 294-306.

32. Tsuda T, Horio F, Uchida K, Aoki H \& Osawa T (2003) Dietary cyanidin 3-O- $\beta$-D-glucoside-rich purple corn color prevents obesity and ameliorates hyperglycemia in mice. J Nutr 133, 2125-2130.

33. Tsuda T, Ueno $\mathrm{Y}$, Aoki H, Koda T, Horio F, Takahashi N, Kawada T \& Osawa T (2004) Anthocyanin enhances adipocytokine secretion and adipocyte-specific gene expression in isolated rat adipocytes. Biochem Biophys Res Comm 316, 149-157. 
34. Jayaprakasam B, Vareed SK, Olsen LK \& Nair MG (2005) Insulin secretion by bioactive anthocyanins and anthocyanidins present in fruits. J Agric Food Chem 53, 28-31.

35. Manach C, Williamson G, Morand C, Scalbert A \& Remesy C (2005) Bioavailability and bioefficacy of polyphenols in humans. I. Review of 97 bioavailability studies. Am J Clin Nutr 81, 230S-242S.

36. Morazzoni P \& Magistretti MJ (1990) Activity of Myrtocyan ${ }^{\circledR}$, an anthocyanoside complex from Vaccinium myrtillus (VMA), on platelet aggregation and adhesiveness. Fitoterapia 61, $13-21$.

37. Hubbard GP, Wolffram S, Lovegrove JA \& Gibbins JM (2003) The role of polyphenolic compounds in the diet as inhibitors of platelet function. Proc Nutr Soc 62, 469-478.

38. Jenkins DJ, Kendall CW, Marchie A, et al. (2005) Direct comparison of a dietary portfolio of cholesterol-lowering foods with a statin in hypercholesterolemic participants. Am J Clin Nutr 81, $380-387$. 